\title{
Reply to: Interobserver variability in colorectal cancer and the 2016 ITBCC consensus
}

\author{
Heather Dawson ${ }^{1} \cdot$ Richard Kirsch $^{2}$ - Alessandro Lugli ${ }^{1}$ \\ on behalf of the participants of the International Tumor Budding Consensus Conference 2016 (1)
}

Received: 19 June 2018 / Accepted: 19 June 2018 / Published online: 2 January 2019

(c) United States \& Canadian Academy of Pathology 2018

We very much appreciate the comments made by Drs. LinoSilva et al. [1] regarding the recommendations of the International Budding Consensus Conference (ITBCC) 2016 [2]. The authors raise several important issues on tumor budding, which we agree need to be clarifieds.

From the data presented by the authors, interobserver variability regarding the exact number of counted buds was fair at most, yet the agreement on budding grade nearly perfect. Adjustment for field size had essentially no effect on the assessment of tumor budding grade. Therefore, the authors suggest that precise methods to score tumor budding as proposed by the ITBCC are unnecessary. Certainly, our colleagues are correct in their statement that tumor budding is such a robust prognostic factor that many different scoring methods as previously proposed in the literature have led to similar results. However, the lack of a standardized method has been one of the major barriers to the routine reporting of tumor budding in pathology practice. Indeed, since publication of the ITBCC recommendations, tumor budding has been incorporated as a recommended element in the CAP colorectal cancer protocol [3].

The importance of evidence-based standardization of tumor budding assessment extends beyond routine diagnostic practice. Without a standardized method of assessment, research studies cannot be validated or compared and tumor budding will not be included in clinical trials or prospective studies. The goal of the ITBCC was therefore to agree on a standardized method supported by the strongest available evidence. As detailed in the ITBCC proceedings, the conference was carefully planned and structured

Heather Dawson

heather.dawson@pathology.unibe.ch

Institute of Pathology, University of Bern, Bern, Switzerland

2 Pathology and Laboratory Medicine, Mount Sinai Hospital, University of Toronto, Toronto, ON, Canada ensuring rigorous assessment of the level of evidence in the literature (according to GRADE) as a basis for formulating recommendations. Core aspects of the Delphi method, namely the definition of topics and questions to be addressed, the selection of an expert panel and a premeeting survey, were fulfilled. Therefore, we believe our approach has integrated the most relevant aspects of assessing and reporting tumor budding according to available data and expert opinion at the time.

As tumor budding is assessed by counting individual buds along the tumor invasive front, it is by nature subject to interobserver variability. While the Kappa statistic is appropriate for evaluating agreement on tumor budding grade, we question its use by the authors for evaluating a continuous variable (exact number of tumor buds) where other methods, such as the intraclass correlation coefficient (ICC) are more appropriate. This could explain the large discrepancy between kappa values for tumor budding grade $(k=0.96)$ and exact number of tumor buds $(k=0.25)$ reported by the authors. Moreover, since clinical management decisions are generally based on tumor budding grade (rather than absolute bud count), the near perfect kappa values for grade reported by the authors is encouraging.

Although it is established that training can improve accuracy and reproducibility of tumor budding assessment $[4,5]$, the exact number of counted buds will likely vary even among expert pathologists, albeit within a narrow range. It is also worth noting that in some instances, a minor difference in bud count can affect assignment of budding grade $(\mathrm{Bd})$. For instance, bud counts of nine and ten are categorized as $\mathrm{Bd} 2$ and $\mathrm{Bd} 3$, respectively, despite minimal differences in biological risk. For this reason, ITBCC strongly recommends the reporting the bud count along with tumor budding grade. The approach of a combined count/cutoff score is intended to facilitate decision-making and still convey where a patient's score lies on a biological spectrum. Finally, it should be emphasized that tumor budding should not be interpreted in isolation, but taken 
into account along with other clinico-pathological factors when making clinical management decisions.

The ITBCC recommends reporting tumor budding in endoscopically resected pT1 CRC and stage II CRC. As noted by the authors, the application of tumor budding in pre-operative rectal cancer biopsies is a third potential scenario where budding may complement staging, as tumor budding in biopsy material has been associated with nodal metastases. However, further studies must examine certain aspects of tumor budding in biopsies, such as the number of biopsies required for evaluation of tumor budding, the assessment of specimen adequacy and the optimum method for tumor bud counting; as such, routine reporting of tumor budding in biopsy specimens is not yet recommended. Finally, the authors question how the accuracy of tumor budding assessment of tumor budding might be improved without adding time and complexity to the evaluation. Training and experience, a clear definition of tumor budding, and awareness of scenarios in which bud counting should be avoided (e.g., glandular fragmentation secondary to heavy inflammation, mucinous pools, etc.) may all help to improve diagnostic accuracy [6]. In special variants of colorectal cancer (e.g., mucinous, signet-ring cell, medullary, and micropapillary), tumor budding should be performed with caution taking care not to count tumor cells suspended in mucin. While tumor budding scores should be adjusted to an area of $0.785 \mathrm{~mm}^{2}$ to ensure standardized reporting, it is impractical to perform mathematical calculations during sign-out. To facilitate this normalization, a conversion table with the number of buds counted and corresponding number of buds to report may be useful.

Clearly, there is much to be learned about tumor budding in solid cancers. The ITBCC is by no means intended to be an end-point, but the rather the basis for standardized assessment of tumor budding in routine diagnostic practice and research according to the most structured and validated available method. It is recommended that tumor budding be factored into clinical management decisions in the context with other clinico-pathologic factors. As data on tumor budding accumulates, the 2016 ITBCC guidelines are open to change if there is good evidence to justify their modification. We also anticipate significant progress in digital image analysis and machine-learning algorithms which may widen our knowledge in the future and possibly improve the objective assessment of tumor budding.

\section{References}

1. Lino-Silva LS, Gamboa-Domínguez A, Zúñiga-Tamayo D, LópezCorrea P. Interobserver variability in colorectal cancer and the 2016 ITBCC concensus. Mod Pathol 2018. https://doi.org/10.1038/ s41379-018-0027-5.

2. Lugli A, Kirsch R, Ajioka Y, Bosman F, Cathomas G, Dawson H, et al. Recommendations for reporting tumor budding in colorectal cancer based on the International Tumor Budding Consensus Conference (ITBCC) 2016. Mod Pathol. 2017;30:1299-311.

3. Kakar SSC, Berho ME, et al. College of American pathologists: protocol for the examination of specimens from patients with primary carcinoma of the colon and rectum. Edition 4.0.0.1. http://www.cap.org/ShowProperty?nodePath=/UCMCon/ Contribution\%20Folders/WebContent/pdf/cp-colon-17protocol4001.pdf.

4. Kai K, Aishima S, Aoki S, Takase Y, Uchihashi K, Masuda M, et al. Cytokeratin immunohistochemistry improves interobserver variability between unskilled pathologists in the evaluation of tumor budding in T1 colorectal cancer. Pathol Int. 2016;66:75-82.

5. Martin B, Schafer E, Jakubowicz E, Mayr P, Ihringer R, Anthuber $\mathrm{M}$, et al. Interobserver variability in the H\&E-based assessment of tumor budding in pT3/4 colon cancer: does it affect the prognostic relevance? Virchows Arch 2018. Apr 6. https://doi.org/10.1007/ s00428-018-2341-1.

6. Koelzer VH, Zlobec I, Berger MD, Cathomas G, Dawson H, Dirschmid K, et al. Tumor budding in colorectal cancer revisited: results of a multicenter interobserver study. Virchows Arch. 2015;466:485-93. 